\title{
Robust Fault Diagnosis Design for Linear Multiagent Systems with Incipient Faults
}

\author{
Jingping Xia, ${ }^{1}$ Bin Jiang, ${ }^{1}$ Ke Zhang, ${ }^{1}$ and Jinfa $\mathrm{Xu}^{2}$ \\ ${ }^{1}$ College of Automation Engineering, Nanjing University of Aeronautics and Astronautics, Nanjing 210016, China \\ ${ }^{2}$ College of Aerospace Engineering, Nanjing University of Aeronautics and Astronautics, Nanjing 210016, China \\ Correspondence should be addressed to Jingping Xia; xiajingping@nuaa.edu.cn
}

Received 30 July 2014; Accepted 24 September 2014

Academic Editor: Peng Shi

Copyright (c) 2015 Jingping Xia et al. This is an open access article distributed under the Creative Commons Attribution License, which permits unrestricted use, distribution, and reproduction in any medium, provided the original work is properly cited.

\begin{abstract}
The design of a robust fault estimation observer is studied for linear multiagent systems subject to incipient faults. By considering the fact that incipient faults are in low-frequency domain, the fault estimation of such faults is proposed for discrete-time multiagent systems based on finite-frequency technique. Moreover, using the decomposition design, an equivalent conclusion is given. Simulation results of a numerical example are presented to demonstrate the effectiveness of the proposed techniques.
\end{abstract}

\section{Introduction}

In the past two decades, the study of multiagent systems has been a very hot topic and attracted considerable attention [1-7]. Multiagent systems appear in various fields, such as cooperative control of unmanned air vehicles and satellite formation flying. However, fault diagnosis is an important problem in automatic control systems and has been an active research area during the past three decades. Fruitful results related to this topic can be found in several excellent works [8-10] and references therein. However, there are very few results about fault diagnosis of multiagents.

In general, according to the varying rate of faults, the faults can be classified into abrupt and incipient in practical systems. Abrupt faults denote system parameter changes faster than the fault-free cases, whose property is able to detect this type of fault quickly to avoid serious consequences, while incipient faults are represented by drift-type changes and are more important in slowly developing problems. Compared with abrupt faults, early fault detection is the key objective for the class of incipient faults. Therefore, the development of effective fault diagnosis schemes for incipient faults is very important. In $[11,12]$, an adaptive online approximation method was proposed to detect incipient faults. And a system decomposition based method was proposed in $[13,14]$ to achieve incipient fault diagnosis, while, in this paper, we consider the problem of fault diagnosis from the perspective of frequency property of incipient faults.

As we know the $H_{\infty}$ performance is the maximum singular value of the transfer function of the studied system. However, if the possible occurring faults are classified into a finitefrequency range, the nominal $H_{\infty}$ design for the entire range will bring much conservatism. For example, incipient faults belong to low-frequency range. Reference [15] not only considered $H_{\infty}$ properties in different frequency domains, but also provided exact linear matrix inequalities (LMIs) characterization in finite-frequency ranges, which can be viewed a useful tool to handle incipient fault diagnosis. Therefore, the issue of incipient fault estimation of discrete-time multiagent systems is a meaningful research and motivates our study.

In this paper, on the basis of existing results [16-18], main contributions of this paper are as follows. According to the frequency range of incipient faults, a fault estimation observer is proposed to achieve incipient fault estimation for multiagent systems using the finite-frequency design. Moreover, an equivalent conclusion of the obtained results is derived by the decomposition technique.

The rest of this paper is organized as follows. Preliminaries and the system description of multiagents have been presented in Section 2. In Section 3, the fault estimation 
observer of multiagents with incipient faults is presented. Simulation results are presented in Section 4 to show the effectiveness of the proposed approach, followed by some conclusions in Section 5.

\section{Preliminaries and Problem Statement}

2.1. Graph Theory. Consider a directed graph $\mathscr{G}=(\mathscr{V}, \mathscr{E}, \mathscr{A})$ with a nonempty finite set of $N$ nodes $\mathscr{V}=\left(v_{1}, v_{2}, \ldots, v_{N}\right)$, a set of edges or arcs $\mathscr{E} \subset \mathscr{V} \times \mathscr{V}$, and the associated adjacency matrix $\mathscr{A}=\left[a_{i j}\right] \in \mathbb{R}^{N \times N}$. In this note, the graph is assumed to be time-invariant; that is, $\mathscr{A}$ is constant. An edge rooted at node $j$ and ended at node $i$ is denoted by $\left(v_{j}, v_{i}\right)$, which means information can flow from node $j$ to node $i . a_{i j}$ is the weight of edge $\left(v_{j}, v_{i}\right)$ and $a_{i j}=1$ if $\left(v_{j}, v_{i}\right) \in \mathscr{E}$; otherwise $a_{i j}=0$. We assume there are no repeated edges and no self-loops, that is, $a_{i i}=0, i \in \mathcal{N}$ with $\mathcal{N}=\{1,2, \ldots, N\}$. Node $j$ is called a neighbor of node $i$ if $\left(v_{j}, v_{i}\right) \in \mathscr{E}$. The set of neighbors of node $i$ is denoted by $N_{i}=\left\{j \mid\left(v_{j}, v_{i}\right) \in \mathscr{E}\right\}$. Define the in-degree matrix as $D=\operatorname{diag}\left\{d_{i}\right\} \in \mathbb{R}^{N \times N}$ with $d_{i}=\sum_{j \in N_{i}} a_{i j}$ and the Laplacian matrix as $L=D-\mathscr{A}$. In this paper, we consider the case of undirected graph.

2.2. Problem Statement. Consider the following multiagent systems with $N$ nodes and a communication graph $\mathscr{G}$ :

$$
\begin{gathered}
x_{i}(k+1)=A x_{i}(k)+B u_{i}(k)+H f_{i}(k)+D_{1} \omega_{i}(k), \\
y_{i}(k)=C x_{i}(k)+D_{2} \omega_{i}(k),
\end{gathered}
$$

where $x_{i}(k) \in \mathbb{R}^{n}$ is the state vector, $u_{i}(k) \in \mathbb{R}^{m}$ is the input vector, $y_{i}(k) \in \mathbb{R}^{p}$ is the output vector, $\omega(k) \in \mathbb{R}^{d}$ is the disturbance and noise which belong to $l_{2}[0,+\infty)$, and $f_{i}(k) \in$ $\mathbb{R}^{r}$ represents the actuator fault. $A, B, H, C, D_{1}$, and $D_{2}$ are constant real matrices of appropriate dimensions. It is supposed that matrices $H$ and $C$ are of full rank. It is supposed that the pair $(A, C)$ is observable.

The dynamics of the leader or control node, labeled 0 , is given by

$$
\begin{gathered}
x_{0}(k+1)=A_{0} x_{0}(k), \\
y_{0}(k)=C_{0} x_{0}(k),
\end{gathered}
$$

where $x_{0}(k) \in \mathbb{R}^{n}$ is the state vector and $y_{0}(k) \in \mathbb{R}^{p}$ is the output vector. It can be considered as a command generator, which generates the desired target trajectory. The leader node can be observed from a small subset of nodes in graph $\mathscr{G}$. If node $i$ observes the leader, an edge $\left(v_{0}, v_{i}\right)$ is said to exist with weighting gain $g_{i}=1$. We refer to node $i$ with $g_{i}=1$ as a pinned or controlled node. Denote the pinning matrix as $G=$ $\operatorname{diag}\left\{g_{i}\right\} \in \mathbb{R}^{N \times N}$.

Lemma 1 (see [15]). For the following linear discrete-time system

$$
\begin{gathered}
\mathscr{X}(k+1)=\mathscr{A} \mathscr{X}(k)+\mathscr{B} \mathcal{U}(k) \\
\mathscr{Y}(k)=\mathscr{C} \mathscr{X}(k)+\mathscr{D} \mathscr{U}(k),
\end{gathered}
$$

its transfer function can be written as $\mathscr{G}\left(e^{j \theta}\right)=\mathscr{C}\left(e^{j \theta} I-\right.$ $\mathscr{A})^{-1} \mathscr{B}+\mathscr{D}$. Given a symmetric matrix $\Pi$, the following two statements are equivalent.

(i) The finite-frequency inequality

$$
\left[\begin{array}{c}
\mathscr{G}\left(e^{j \theta}\right) \\
\mathscr{I}
\end{array}\right]^{T} \Pi\left[\begin{array}{c}
\mathscr{G}\left(e^{j \theta}\right) \\
\mathscr{I}
\end{array}\right]<0
$$

(ii) There exist Hermitian matrices $\mathscr{P}$ and $\mathcal{Q}$ (where $Q>$ $0)$, and

$$
\left[\begin{array}{cc}
\mathscr{A} & \mathscr{B} \\
\mathscr{I} & 0
\end{array}\right]^{T} \Xi\left[\begin{array}{cc}
\mathscr{A} & \mathscr{B} \\
\mathscr{I} & 0
\end{array}\right]+\left[\begin{array}{cc}
\mathscr{C} & \mathscr{D} \\
0 & \mathscr{I}
\end{array}\right]^{T} \Pi\left[\begin{array}{cc}
\mathscr{C} & \mathscr{D} \\
0 & \mathscr{I}
\end{array}\right]<0
$$

where

$$
\Xi=\left[\begin{array}{cc}
\mathscr{P} & \mathscr{Q} \\
\mathscr{Q} & -\mathscr{P}-2 \cos \left(\vartheta_{l}\right) \mathscr{Q}
\end{array}\right]
$$

for low-frequency range $|\vartheta| \leq \vartheta_{l}$, and

$$
\Xi=\left[\begin{array}{cc}
\mathscr{P} & -\mathscr{Q} \\
-\mathscr{Q} & -\mathscr{P}+2 \cos \left(\vartheta_{h}\right) \mathbb{Q}
\end{array}\right]
$$

for high-frequency range $|\vartheta| \geq \vartheta_{h}$.

\section{Main Results}

For the dynamics (1), the fault estimation observer of the $i$ th node is constructed:

$$
\begin{gathered}
\widehat{x}_{i}(k+1)=A \widehat{x}_{i}(k)+B u_{i}(k)+H \widehat{f}_{i}(k)-R \zeta_{i}(k), \\
\hat{y}_{i}(k)=C \widehat{x}_{i}(k), \\
\widehat{f}_{i}(k+1)=\widehat{f}_{i}(k)-F \zeta_{i}(k),
\end{gathered}
$$

where $\widehat{x}_{i}(k) \in \mathbb{R}^{n}$ is the observer state, $\widehat{y}_{i}(k) \in \mathbb{R}^{p}$ is the observer output, $\hat{f}_{i}(k) \in \mathbb{R}^{r}$ is an estimate of $f_{i}(k)$, and $R \in$ $\mathbb{R}^{n \times p}, F \in \mathbb{R}^{r \times p}$ are observer gain matrices of node $i . \zeta_{i}(k)$ is the neighborhood output estimation error of the $i$ th node, that is,

$$
\begin{aligned}
\zeta_{i}(k)= & \sum_{j \in N_{i}} a_{i j}\left(\left(\widehat{y}_{i}(k)-y_{i}(k)\right)-\left(\widehat{y}_{j}(k)-y_{j}(k)\right)\right) \\
& +g_{i}\left(\left(\widehat{y}_{i}(k)-y_{i}(k)\right)-\left(\widehat{y}_{0}(k)-y_{0}(k)\right)\right) .
\end{aligned}
$$

Remark 2. Here, it is assumed that the state of leader mode is measured or available, so the observed output obtained from the observer is equal to the measured output, that is, $\widehat{y}_{0}(k)-$ $y_{0}(k)=0$.

For the $i$ th node, let

$$
\begin{gathered}
e_{x i}(k)=\widehat{x}_{i}(k)-x_{i}(k), \quad e_{y i}(k)=\widehat{y}_{i}(k)-y_{i}(k), \\
e_{f i}(k)=\widehat{f}_{i}(k)-f_{i}(k) ;
\end{gathered}
$$


one gets

$$
\begin{aligned}
& e_{x i}(k+1) \\
& =A e_{x i}(k)+H e_{f i}(k) \\
& -R\left[\sum_{j \in N_{i}} a_{i j}\left(C e_{x i}(k)-D_{2} \omega_{i}(k)-C e_{x j}(k)+D_{2} \omega_{j}(k)\right)\right. \\
& \left.+g_{j}\left(C e_{x i}(k)-D_{2} \omega_{i}(k)\right)\right]-D_{1} \omega_{i}(k), \\
& e_{f i}(k+1) \quad \begin{array}{l}
e_{f i}(k) \\
-F\left[\sum_{j \in N_{i}} a_{i j}\left(C e_{x i}(k)-D_{2} \omega_{i}(k)-C e_{x j}(k)+D_{2} \omega_{j}(k)\right)\right.
\end{array} \\
& \left.+g_{j}\left(C e_{x i}(k)-D_{2} \omega_{i}(k)\right)\right]-\Delta f_{i}(k),
\end{aligned}
$$

where $\Delta f_{i}(k)=f_{i}(k+1)-f_{i}(k)$.

Based on (11), the following augmented system can be obtained:

$$
\begin{aligned}
& {\left[\begin{array}{l}
e_{x i}(k+1) \\
e_{f i}(k+1)
\end{array}\right]} \\
& =\left[\begin{array}{cc}
A & H \\
0 & I
\end{array}\right]\left[\begin{array}{l}
e_{x i}(k) \\
e_{f i}(k)
\end{array}\right]-\left[\begin{array}{l}
R \\
F
\end{array}\right] \\
& \quad \times\left[\sum _ { j \in N _ { i } } a _ { i j } \left(C e_{x i}(k)-D_{2} \omega_{i}(k)-C e_{x j}(k)\right.\right. \\
& \left.\quad+D_{2} \omega_{j}(k)\right)+g_{j}\left(C e_{x i}(k)-D_{2} \omega_{i}(k)\right) \\
& -\left[\begin{array}{c}
D_{1} \\
0
\end{array}\right] \omega_{i}(k)-\left[\begin{array}{l}
0 \\
I
\end{array}\right] \Delta f_{i}(k) .
\end{aligned}
$$

Furthermore, denote new vectors and matrices:

$$
\begin{gathered}
\bar{e}_{i}(k)=\left[\begin{array}{c}
e_{x i}(k) \\
e_{f i}(k)
\end{array}\right], \quad v_{i}(k)=\left[\begin{array}{c}
\omega_{i}(k) \\
\Delta f_{i}(k)
\end{array}\right], \\
\bar{A}=\left[\begin{array}{cc}
A & H \\
0 & I
\end{array}\right], \quad \bar{R}=\left[\begin{array}{l}
R \\
F
\end{array}\right], \quad \bar{C}=\left[\begin{array}{ll}
C & 0
\end{array}\right], \\
\bar{D}_{1}=\left[\begin{array}{c}
D_{1} \\
0
\end{array}\right], \quad \bar{I}_{r}=\left[\begin{array}{l}
0 \\
I
\end{array}\right] ;
\end{gathered}
$$

then for the $i$ th node, it follows that

$$
\begin{aligned}
& \bar{e}_{i}(k+1) \\
& =\bar{A} \bar{e}_{i}(k) \\
& -\bar{R}\left[\sum_{j \in N_{i}} a_{i j}\left(\bar{C}^{-} e_{i}(k)-D_{2} \omega_{i}(k)-\bar{C} e_{j}(k)+D_{2} \omega_{j}(k)\right)\right. \\
& \left.+g_{j}\left(\bar{C} \bar{e}_{i}(k)-D_{2} \omega_{i}(k)\right)\right]-\bar{D}_{1} \omega_{i}(k)-\bar{I}_{r} \Delta f_{i}(k) \\
& e_{f i}(k)=\bar{I}_{r}^{T} \bar{e}_{i}(k) .
\end{aligned}
$$

Denote the global variable

$$
\begin{aligned}
\bar{e}(k) & =\left[\bar{e}_{1}^{T}(k), \bar{e}_{2}^{T}(k), \ldots, \bar{e}_{N}^{T}(k)\right]^{T}, \\
e_{f}(k) & =\left[e_{f 1}^{T}(k), e_{f 2}^{T}(k), \ldots, e_{f N}^{T}(k)\right]^{T}, \\
\omega(k) & =\left[\omega_{1}^{T}(k), \omega_{2}^{T}(k), \ldots, \omega_{N}^{T}(k)\right]^{T}, \\
\Delta f(k) & =\left[\Delta f_{1}^{T}(k), \Delta f_{2}^{T}(k), \ldots, \Delta f_{N}^{T}(k)\right]^{T} ;
\end{aligned}
$$

then the global error dynamics can be expressed as

$$
\begin{aligned}
\bar{e}(k+1)= & \left(I_{N} \otimes \bar{A}\right) \bar{e}(k)-\left(I_{N} \otimes \bar{R}\right) \\
& \times\left[(L+G) \otimes \bar{C} \bar{e}(k)-(L+G) \otimes D_{2} \omega(k)\right] \\
& -\left(I_{N} \otimes \bar{D}_{1}\right) \omega(k)-\left(I_{N} \otimes \bar{I}_{r}\right) \Delta f(k) \\
= & {\left[\left(I_{N} \otimes \bar{A}\right)-(L+G) \otimes(\overline{R C})\right] \bar{e}(k) } \\
& +\left[(L+G) \otimes\left(\bar{R} D_{2}\right)-\left(I_{N} \otimes \bar{D}_{1}\right)\right] \omega(k) \\
& -\left(I_{N} \otimes \bar{I}_{r}\right) \Delta f(k), \\
& e_{f}(k)=\left(I_{N} \otimes \bar{I}_{r}^{T}\right) \bar{e}(k),
\end{aligned}
$$

where $\otimes$ denotes Kronecker product [19]

Theorem 3. Given scalars $\gamma_{1}, \gamma_{2}>0$. The global error dynamics (16) satisfy the $H_{\infty}$ performances $\left\|T_{\omega(k) e_{f}(k)}\right\|_{\infty}<$ $\gamma_{1}$ and $\left\|T_{\Delta f(k) e_{f}(k)}\right\|_{\infty}<\gamma_{2}$ if there exist symmetric positive definite matrices $\bar{Q}_{1}, \bar{Q}_{2}$, symmetric matrices $\bar{P}_{1}, \bar{P}_{2}$, and matrices $\bar{S}, \bar{Y}$ such that the following conditions hold: 


$$
\begin{gathered}
{\left[\begin{array}{cccc}
I_{N} \otimes\left(-\bar{S}-\bar{S}^{T}+\bar{P}_{1}\right) & I_{N} \otimes\left(\bar{Q}_{1}+\overline{S A}\right)-(L+G) \otimes(\overline{Y C}) & \phi & 0 \\
* & I_{N} \otimes\left(-2 \cos \left(\theta_{f}\right) \bar{Q}_{1}-\bar{P}_{1}\right) & 0 & I_{N} \otimes \bar{I}_{r} \\
* & * & -\gamma_{1} I & 0 \\
* & * & * & -\gamma_{1} I
\end{array}\right]<0,} \\
{\left[\begin{array}{cccc}
I_{N} \otimes\left(-\bar{S}-\bar{S}^{T}+\bar{P}_{2}\right) & I_{N} \otimes\left(-\bar{Q}_{2}+\overline{S A}\right)-(L+G) \otimes(\overline{Y C}) & \varphi & 0 \\
* & I_{N} \otimes\left(2 \cos \left(\theta_{d}\right) \bar{Q}_{2}-\bar{P}_{2}\right) & 0 & I_{N} \otimes \bar{I}_{r} \\
* & * & -\gamma_{2} I & 0 \\
* & * & * & -\gamma_{2} I
\end{array}\right]<0,}
\end{gathered}
$$

where $\phi=(L+G) \otimes\left(\bar{Y} D_{2}\right)-I_{N} \otimes\left(\overline{S D}_{1}\right)$ and $\varphi=-I_{N} \otimes\left(\overline{S I}_{r}\right)$; then the fault estimation observer gain matrix is given by $\bar{R}=$ $\bar{S}^{-1} \bar{Y}$.

Remark 4. In general, the noises are in high-frequency domain and incipient faults are in low-frequency one. So according to [17] and Lemma 1, the proof of Theorem 3 can be deduced by choosing symmetric positive definite matrices $I_{N} \otimes \bar{Q}_{1}, I_{N} \otimes \bar{Q}_{2}$, symmetric matrices $I_{N} \otimes \bar{P}_{1}, I_{N} \otimes \bar{P}_{2}$, and a matrix $I_{N} \otimes \bar{S}, I_{N} \otimes \bar{Y}$. For brevity, this proof is not provided here.

Remark 5. From the process of fault estimation observer design, we can see that there is no parameter value of leader node 0 because of the assumption that the state of leader node is measurable, as shown in Remark 2. Since undirected graph is considered in this paper, matrix $L$ is symmetric such that one of the eigenvalues of $L$ is zero, and the others are positive. Therefore, the presented leader node can be viewed as a virtual node, whose function is to make matrix $(L+G)$ nonsingular.

Remark 6. From [20], we can get the matrix $(L+G)$ is nonsingular. Furthermore, based on the decomposition design in [21], an equivalent conclusion of Theorem 3 is obtained, that is, Theorem 7.

Theorem 7. Given scalars $\gamma_{1}, \gamma_{2}>0$. The global error dynamics (16) satisfy the $H_{\infty}$ performances $\left\|T_{\omega(k) e_{f}(k)}\right\|_{\infty}<\gamma_{1}$ and $\left\|T_{\Delta f(k) e_{f}(k)}\right\|_{\infty}<\gamma_{2}$ if there exist symmetric positive definite matrices $\bar{Q}_{1}, \bar{Q}_{2}$, symmetric matrices $\bar{P}_{1}, \bar{P}_{2}$, and matrices $\bar{S}, \bar{Y}$ such that the following conditions hold:

$$
\begin{gathered}
{\left[\begin{array}{cccc}
-\bar{S}-\bar{S}+\bar{P}_{1} & \bar{Q}_{1}+\overline{S A}-\lambda_{i}(\overline{Y C}) & \lambda_{i}\left(\bar{Y} D_{2}\right)-\overline{S D}_{1} & 0 \\
* & -2 \cos \left(\theta_{f}\right) \bar{Q}_{1}-\bar{P}_{1} & 0 & \bar{I}_{r} \\
* & * & -\gamma_{1} I & 0 \\
* & * & * & -\gamma_{1} I
\end{array}\right]<0, \quad i=1, \ldots, N,} \\
{\left[\begin{array}{cccc}
-\bar{S}-\bar{S}+\bar{P}_{2} & -\bar{Q}_{2}+\overline{S A}-\lambda_{i}(\overline{Y C}) & -\overline{S I}_{r} & 0 \\
* & 2 \cos \left(\theta_{d}\right) \bar{Q}_{2}-\bar{P}_{2} & 0 & \bar{I}_{r} \\
* & * & -\gamma_{2} I & 0 \\
* & * & * & -\gamma_{2} I
\end{array}\right]<0, \quad i=1, \ldots, N,}
\end{gathered}
$$

where $\lambda_{i}(i=1, \ldots, N)$ are the eigenvalues of $(L+G)$ and the fault estimation observer gain matrix is given by $\bar{R}=\bar{S}^{-1} \bar{Y}$.

Proof. Since matrix $(L+G)$ is symmetric positive definite, there always exists a nonsingular matrix $V \in \mathbb{R}^{N \times N}$ such that $V^{T}(L+G) V=\Lambda$ and $V^{T} V=I$, where $\Lambda=\operatorname{diag}\left(\lambda_{1}, \lambda_{2}\right.$, $\left.\ldots, \lambda_{N}\right) \in \mathbb{R}^{N \times N}$ and $\lambda_{1}, \lambda_{2}, \ldots, \lambda_{N}$ are positive. Under the coordinate transformation

$$
\begin{aligned}
\bar{e}(k) & =\left(V \otimes I_{n+r}\right) \breve{\bar{e}}(k), & & \omega(k)=\left(V \otimes I_{d}\right) \breve{\omega}(k), \\
\Delta f(k) & =\left(V \otimes I_{r}\right) \breve{\Delta f}(k), & & \bar{e}_{f}(k)=\left(V \otimes I_{r}\right) \check{\bar{e}}_{f}(k) .
\end{aligned}
$$

And let

$$
\begin{gathered}
\breve{\bar{e}}(k)=\left[\breve{\bar{e}}_{1}^{T}(k), \breve{\bar{e}}_{2}^{T}(k), \ldots, \breve{\bar{e}}_{N}^{T}(k)\right]^{T}, \\
\breve{\omega}(k)=\left[\breve{\omega}_{1}^{T}(k), \breve{\omega}_{2}^{T}(k), \ldots, \breve{\omega}_{N}^{T}(k)\right]^{T}, \\
\breve{\Delta f}(k)=\left[\breve{\Delta f}_{1}^{T}(k), \breve{\Delta f}_{2}^{T}(k), \ldots, \breve{\Delta f}_{N}^{T}(k)\right]^{T}, \\
\breve{e}_{f}(k)=\left[\breve{e}_{f 1}^{T}(k), \breve{e}_{f 2}^{T}(k), \ldots, \breve{e}_{f N}^{T}(k)\right]^{T} ;
\end{gathered}
$$


then it gets

$$
\begin{aligned}
\check{\bar{e}}(k+1) \\
=\left[\left(I_{N} \otimes \bar{A}\right)-\Lambda \otimes(\overline{R C})\right] \breve{\bar{e}}(k) \\
+\left[\Lambda \otimes\left(\bar{R} D_{2}\right)-\left(I_{N} \otimes \bar{D}_{1}\right)\right] \breve{\omega}(k)-\left(I_{N} \otimes \bar{I}_{r}\right) \breve{\Delta f}(k), \\
\breve{e}_{f}(k)=\left(I_{N} \otimes \bar{I}_{r}^{T}\right) \breve{\bar{e}}(k) .
\end{aligned}
$$

Since $\Lambda$ is a diagonal matrix, the transfer function

$$
\begin{aligned}
T_{\breve{\omega}(k) \breve{e}_{f}(k)} & =\operatorname{diag}\left(T_{\breve{\omega}_{1}(k) \breve{e}_{f 1}(k), \ldots, \breve{\omega}_{N}(k) \breve{e}_{f N}(k)}\right) \\
& =\left(V^{-1} \otimes I_{d}\right) T_{\omega(k) e_{f}(k)}\left(V \otimes I_{r}\right), \\
T_{\breve{\Delta f(k) \check{e}_{f}(k)}} & =\operatorname{diag}\left(T_{\breve{\Delta f}_{1}(k) \breve{e}_{f 1}(k), \ldots, \breve{\Delta f}_{N}(k) \breve{e}_{f N}(k)}\right) \\
& =\left(V^{-1} \otimes I_{r}\right) T_{\Delta f(k) e_{f}(k)}\left(V \otimes I_{r}\right),
\end{aligned}
$$

where $V$ is orthogonal. It follows that

$$
\begin{aligned}
& \left\|T_{\breve{\omega}(k) \breve{e}_{f}(k)}\right\|_{\infty}=\max _{i=1, \ldots, N}\left\|T_{\breve{\omega}_{i}(k) \breve{e}_{f i}(k)}\right\|_{\infty}=\left\|T_{\omega(k) e_{f}(k)}\right\|_{\infty}, \\
& \left\|T_{\breve{\Delta f}(k) \breve{e}_{f}(k)}\right\|_{\infty}=\max _{i=1, \ldots, N}\left\|T_{\breve{\Delta f_{i}}(k) \breve{e}_{f i}(k)}\right\|_{\infty}=\left\|T_{\Delta f(k) e_{f}(k)}\right\|_{\infty} .
\end{aligned}
$$

Moreover, by choosing the same matrix variables $\bar{Q}_{1}, \bar{Q}_{2}, \bar{P}_{1}$, $\bar{P}_{2}, \bar{S}, \bar{Y}$ shown in Theorem 3 , one gets the two conditions of Theorems 3 and 7 are equivalent.

Remark 8. Theorem 7 is an equivalent conclusion of Theorem 3 by using decomposition technique. And note that the eigenvalues of matrix $(L+G)$ are positive; that is, $\lambda_{i}>0$, $i=1, \ldots, N$.

\section{Simulation Results}

In this section, the following example is presented to illustrate the effectiveness of the proposed method. It is assumed that there are four agents of multiagent systems, that is, $N=4$, and each node is

$$
\begin{gathered}
x_{i}(k+1)=\left[\begin{array}{cc}
0.1 & -0.1 \\
0.2 & 0.5
\end{array}\right] x_{i}(k)+\left[\begin{array}{c}
1.2 \\
1
\end{array}\right] u_{i}(k), \\
y_{i}(k)=\left[\begin{array}{ll}
1 & 1
\end{array}\right] x_{i}(k) .
\end{gathered}
$$

The disturbance matrices are $D_{1}=\left[\begin{array}{ll}0.1 & 0.1\end{array}\right]^{T}$ and $D_{1}=0.1$. Here, actuator faults are considered, that is, the fault distribution matrix $H=B$.

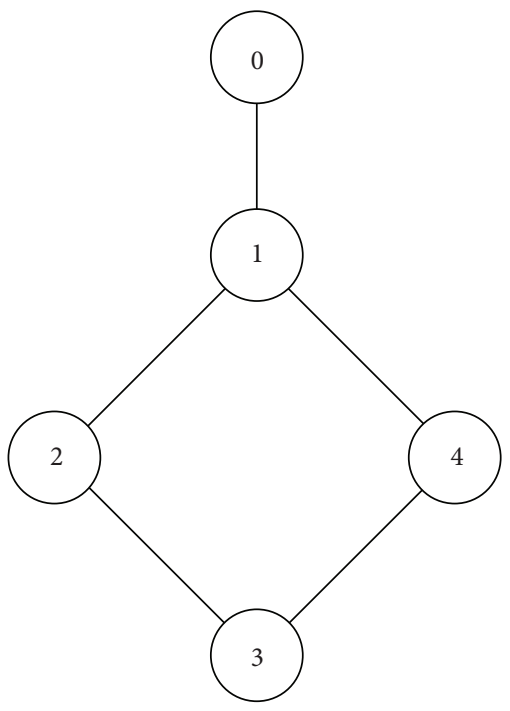

Figure 1: The communication topology of multiagent systems.

For these multiagent systems subject to four nodes, we assume that each node only contacts with its nearest neighbors, as shown in Figure 1. From Figure 1, one gets Laplacian matrix

$$
L=\left[\begin{array}{cccc}
2 & -1 & 0 & -1 \\
-1 & 2 & -1 & 0 \\
0 & -1 & 2 & -1 \\
-1 & 0 & -1 & 2
\end{array}\right]
$$

And only the first node links the leader node, so we have

$$
G=\left[\begin{array}{llll}
1 & 0 & 0 & 0 \\
0 & 0 & 0 & 0 \\
0 & 0 & 0 & 0 \\
0 & 0 & 0 & 0
\end{array}\right] .
$$

It is readily verified that $(L+G)$ is nonsingular and its eigenvalues are positive, that is, $0.1864,2.0000,2.4707$, and 4.3429.

It is assumed that the noise is in high-frequency domain $\vartheta_{d}=\pi \mathrm{rad}$, and the derivative of actuator faults is in lowfrequency domain $\vartheta_{f}=\pi / 10 \mathrm{rad}$.

By solving the conditions of Theorem 3 , one gets $\gamma_{1}+\gamma_{2}=$ 4.9257 and the observer matrix

$$
\bar{R}=\left[\begin{array}{l}
0.0873 \\
0.2476 \\
0.0728
\end{array}\right]
$$

And we are able to verify that the minimum value is also $\gamma_{1}+$ $\gamma_{2}=4.9257$ by calculating the conditions of Theorem 7 .

For simulation, we firstly consider an incipient fault occurring in the first node as follows:

$$
f_{1}(k)= \begin{cases}0, & 0 \mathrm{~s} \leq t<50 \mathrm{~s} \\ 1-e^{-0.05(t-50)}, & 50 \mathrm{~s} \leq t\end{cases}
$$



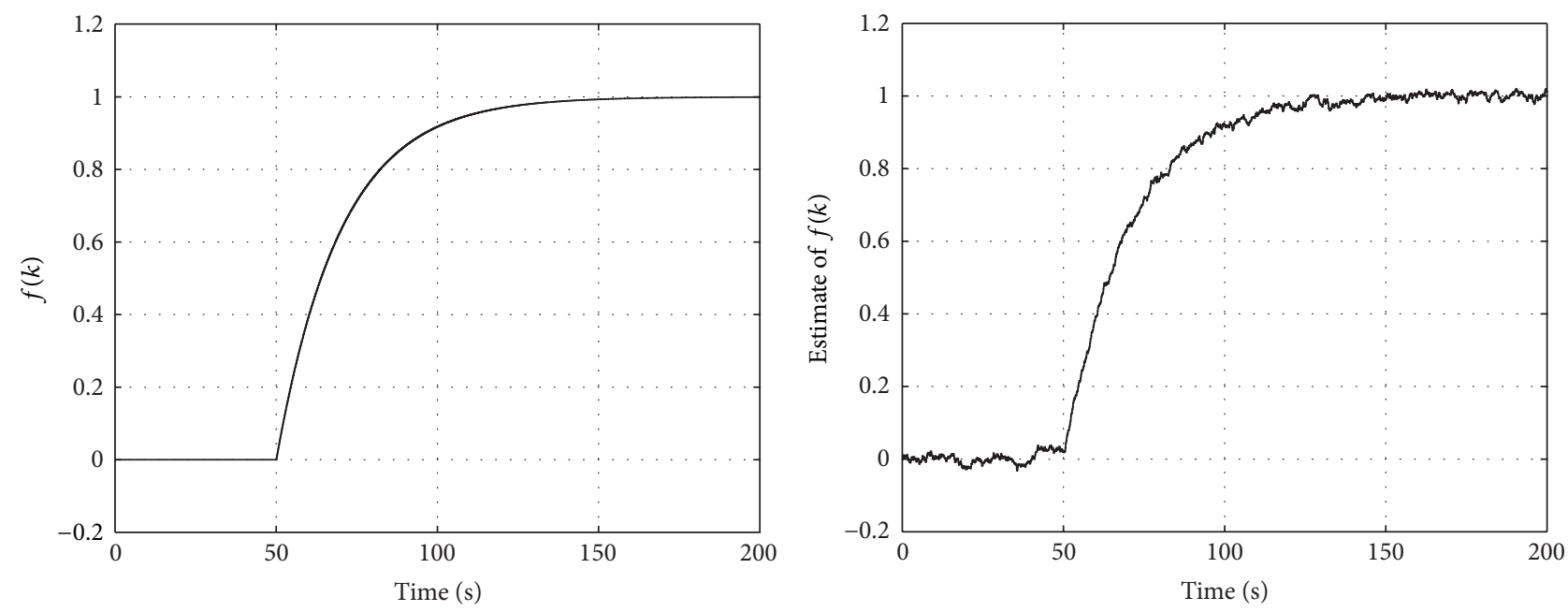

FIGURE 2: Simulation of the incipient fault in the first node.
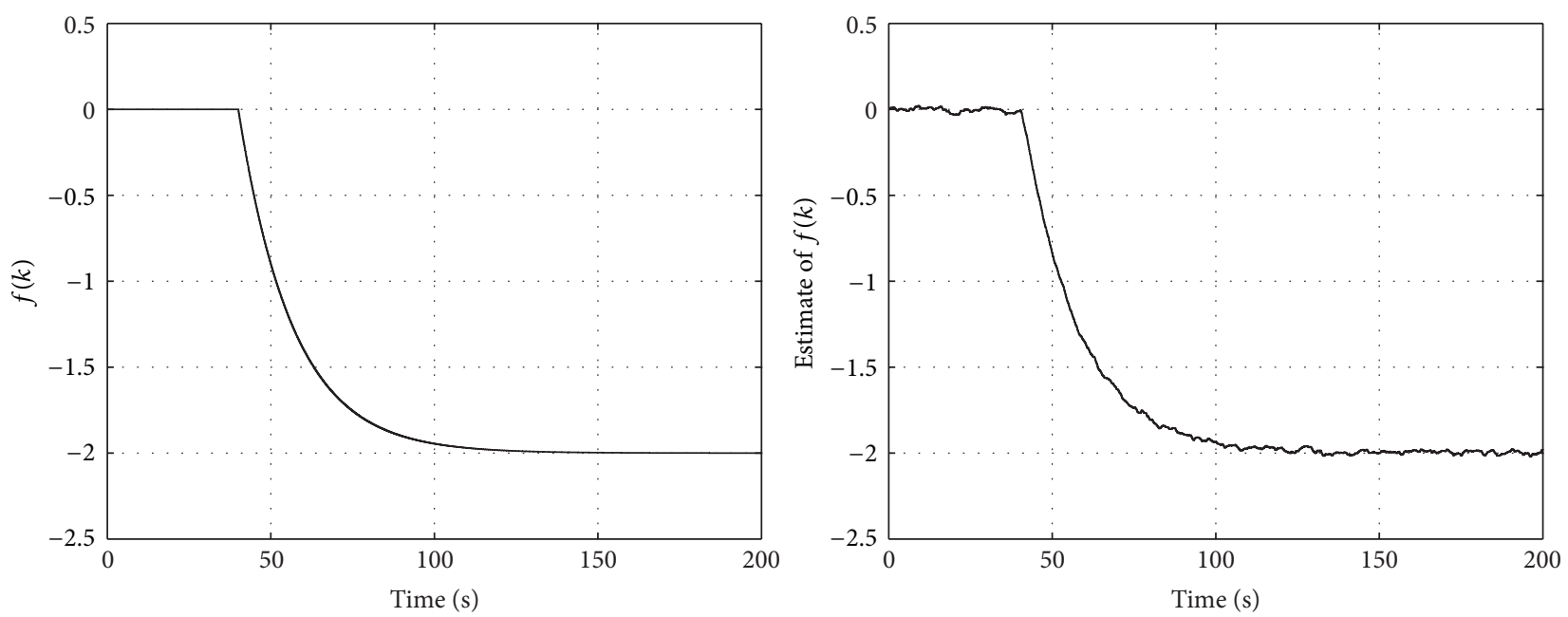

FIGURE 3: Simulation of the incipient fault in the third node.

Simulation result of this fault in the first node is shown in Figure 2.

Secondly, we assume that there is an incipient fault in the third node:

$$
f_{3}(k)= \begin{cases}0, & 0 \mathrm{~s} \leq t<40 \mathrm{~s}, \\ -2\left(1-e^{-0.06(t-40)}\right), & 40 \mathrm{~s} \leq t .\end{cases}
$$
tion.

For such fault, Figure 3 illustrates the online fault estima-

From simulation results, it is concluded that the presented fault estimation observer can achieve incipient fault estimation of multiagent systems.

\section{Conclusions}

In this paper, a fault estimation design has been proposed for a class of multiagent systems with incipient faults. According to the frequency properties of incipient faults and noises, a robust $H_{\infty}$ design with finite-frequency domain is obtained in terms of linear matrix inequalities. An equivalent result of the proposed fault estimation is further presented. Finally, simulation results of incipient faults in the two different nodes have been given to illustrate the effectiveness of the proposed design. Fault diagnosis of nonlinear multiagent systems will be studied in our future work.

\section{Conflict of Interests}

The authors declare that there is no conflict of interests regarding the publication of this paper.

\section{Acknowledgments}

This work is partially supported by the National Natural Science Foundation of China $(61304112$, 61428303), Natural Science Foundation of Jiangsu Province (BK20131364), China Postdoctoral Science Foundation Funded Project (2012M521079, 2013T60535), and Jiangsu Postdoctoral Science Foundation (1201013B). 


\section{References}

[1] Y. Hong, G. Chen, and L. Bushnell, "Distributed observers design for leader-following control of multi-agent networks," Automatica, vol. 44, no. 3, pp. 846-850, 2008.

[2] C.-Q. Ma and J.-F. Zhang, "Necessary and sufficient conditions for consensusability of linear multi-agent systems," IEEE Transactions on Automatic Control, vol. 55, no. 5, pp. 1263-1268, 2010.

[3] Z. Li, Z. Duan, and G. Chen, "Dynamic consensus of linear multi-agent systems," IET Control Theory and Applications, vol. 5, no. 1, pp. 19-28, 2011.

[4] H. Du, S. Li, and P. Shi, "Robust consensus algorithm for second-order multi-agent systems with external disturbances," International Journal of Control, vol. 85, no. 12, pp. 1913-1928, 2012.

[5] Y. Liu and Y. Jia, " $H_{\infty}$ consensus control for multi-agent systems with linear coupling dynamics and communication delays," International Journal of Systems Science, vol. 43, no. 1, pp. 5062, 2012.

[6] M. H. Nguyen and D. Q. Tran, "A combination trust model for multi-agent systems," International Journal of Innovative Computing, Information and Control, vol. 9, no. 6, pp. 2405-2420, 2013.

[7] A. Sedziwy, "Effective graph representation supporting multiagent distributed computing," International Journal of Innovative Computing, Information and Control, vol. 10, no. 1, pp. 101113, 2014.

[8] M. Blanke, M. Kinnaert, J. Lunze, and M. Staroswiecki, Diagnosis and Fault-Tolerant Control, Springer, New York, NY, USA, 2006.

[9] S. X. Ding, Model-Based Fault Diagnosis Techniques: Design Schemes, Algorithms, and Tools, Springer, Berlin, Germany, 2008.

[10] K. Zhang, B. Jiang, and P. Shi, Observer-Based Fault Estimation and Accommodation for Dynamic Systems, Springer, Berlin, Germany, 2013.

[11] M. A. Demetriou and M. M. Polycarpou, "Incipient fault diagnosis of dynamical systems using online approximators," IEEE Transactions on Automatic Control, vol. 43, no. 11, pp. 1612-1617, 1998.

[12] X. Zhang, M. M. Polycarpou, and T. Parisini, "A robust detection and isolation scheme for abrupt and incipient faults in nonlinear systems," IEEE Transactions on Automatic Control, vol. 47, no. 4, pp. 576-593, 2002.

[13] W. Chen and F. N. Chowdhury, "Analysis and detection of incipient faults in post-fault systems subject to adaptive faulttolerant control," International Journal of Adaptive Control and Signal Processing, vol. 22, no. 9, pp. 815-832, 2008.

[14] W. Chen and F. N. Chowdhury, "A synthesized design of slidingmode and Luenberger observers for early detection of incipient faults," International Journal of Adaptive Control and Signal Processing, vol. 24, no. 12, pp. 1021-1035, 2010.

[15] T. Iwasaki and S. Hara, "Generalized KYP lemma: unified frequency domain inequalities with design applications," IEEE Transactions on Automatic Control, vol. 50, no. 1, pp. 41-59, 2005.

[16] K. Zhang, B. Jiang, and P. Shi, "Observer-based integrated robust fault estimation and accommodation design for discretetime systems," International Journal of Control, vol. 83, no. 6, pp. 1167-1181, 2010.
[17] K. Zhang, B. Jiang, P. Shi, and J. Xu, "Fault estimation observer design for discrete-time systems in finite-frequency domain," International Journal of Robust and Nonlinear Control, 2014.

[18] Q. Shen, B. Jiang, P. Shi, and J. Zhao, "Cooperative adaptive fuzzy tracking control for networked unknown nonlinear multi-agent systems with time-varying actuator faults," IEEE Transactions on Fuzzy Systems, vol. 22, no. 3, pp. 494-504, 2014.

[19] H. Zhang, F. L. Lewis, and A. Das, "Optimal design for synchronization of cooperative systems: state feedback, observer and output feedback," IEEE Transactions on Automatic Control, vol. 56, no. 8, pp. 1948-1952, 2011.

[20] Z. Li, Z. Duan, and G. Chen, " $H_{\infty}$ and $H_{2}$ performance regions of multi-agent systems," Automatica, vol. 47, no. 4, pp. 797-803, 2011.

[21] R. Ghadami and B. Shafai, "Decomposition-based distributed control for continuous-time multi-agent systems," IEEE Transactions on Automatic Control, vol. 58, no. 1, pp. 258-264, 2013. 


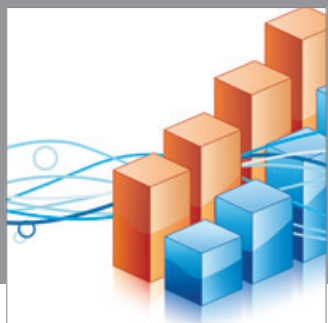

Advances in

Operations Research

mansans

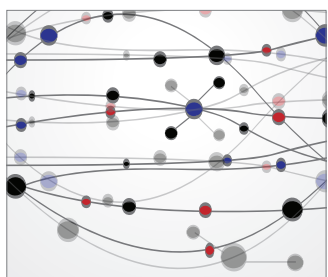

The Scientific World Journal
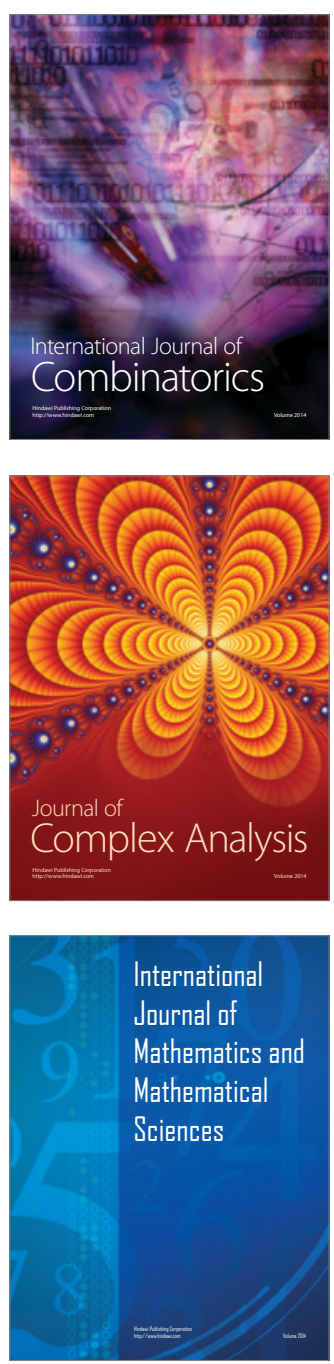
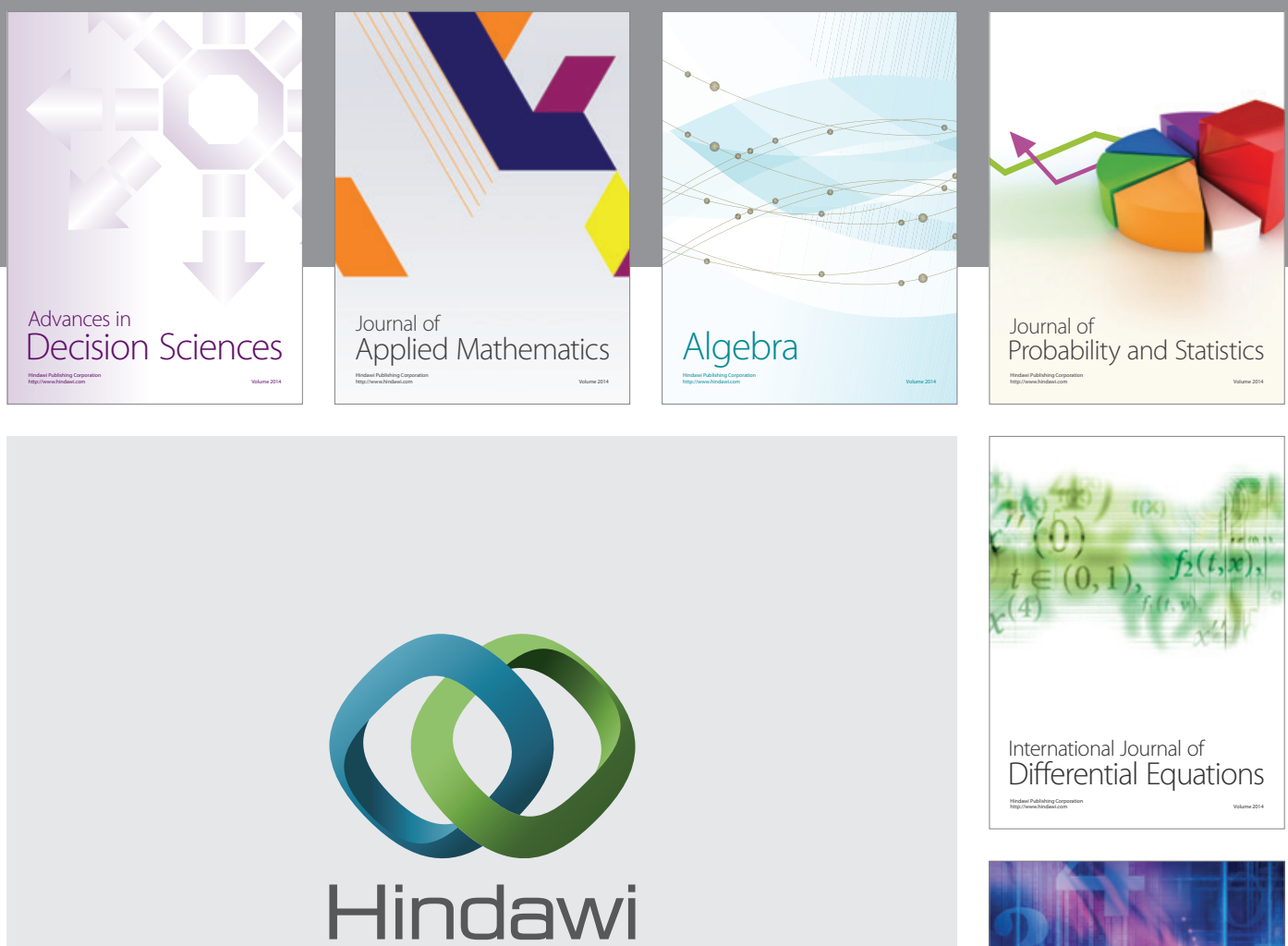

Submit your manuscripts at http://www.hindawi.com
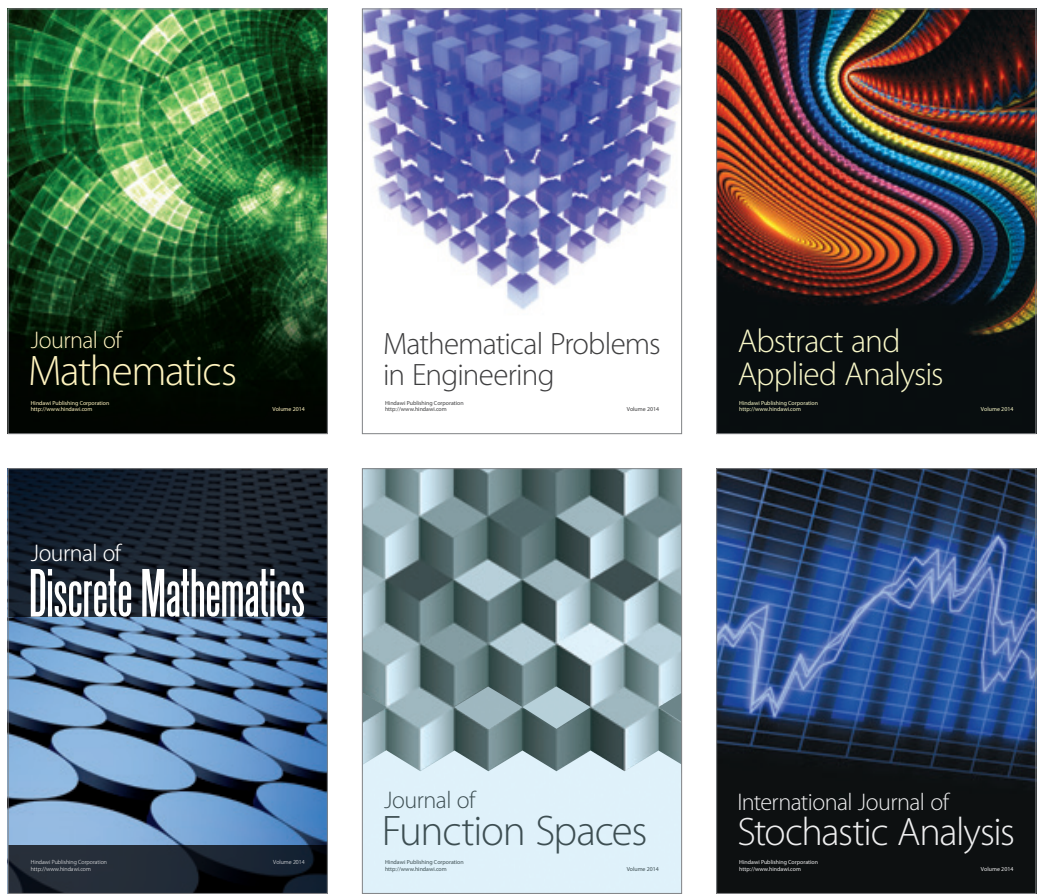

Journal of

Function Spaces

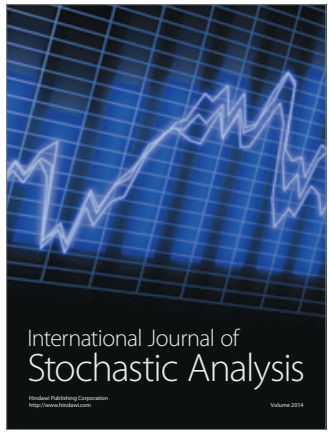

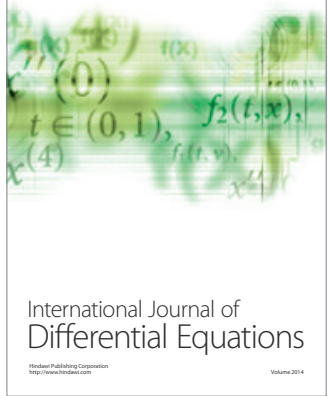
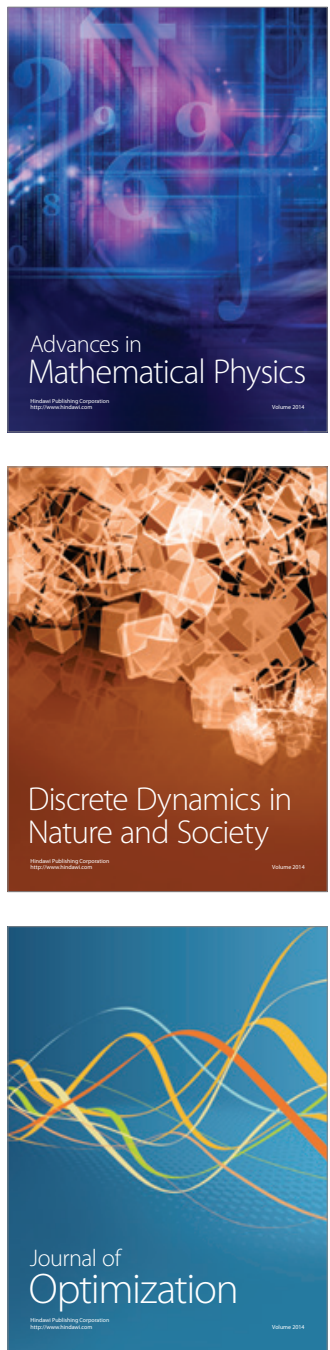